\title{
De la typologie des outils numériques dans le champ des EIAH à leur opérationnalité inclusive
}

\author{
Hervé BENOIT \\ Maître de conférences en sciences de l'éducation \\ INS HEA (Suresnes) - Université Paris Lumières \\ Chercheur au Grhapes (EA 7287)
}

Sylviane FEUILLADIEU

Maître de conférences en sociologie Aix-Marseille Université, ADEF (EA 4671)

Résumé: Dans le champ des Environnements informatiques pour l'apprentissage humain (EIAH), de nombreuses ressources numériques sont aujourd'hui à la disposition des enseignants pour faciliter la transmission des savoirs en général et pour répondre en particulier aux besoins d'élèves en situation de handicap ou de difficulté. L'objet de cette recherche est d'interroger l'opérationnalité inclusive de ces ressources, c'est-à-dire leur capacité à contribuer à l'accessibilité pédagogique universelle. À cette fin, la méthode choisie a consisté à construire un modèle d'analyse typologique sous la forme d'un schéma heuristique destiné à cartographier, à l'aide d'un repère constitué par le croisement de deux axes, celui de la didactisation et celui de la compensation/accessibilité, l'espace conceptuel d'élaboration et de développement de ces outils. Leur visée peut être soit spécifique, soit générique; focalisée sur la suppléance de déficiences ou sur l'optimisation de l'apprentissage pour tous. La localisation sur la carte heuristique de quelques outils numériques au regard de leurs enjeux et de leurs logiques internes, permet de faire émerger l'axe dynamique de la mesure de leur opérationnalité inclusive, transversal à l'espace structuré par les deux précédents.

Mots-clés: Accessibilité - Compensation - Didactisation - EIAH - Handicap - Opérationnalité inclusive Ressources numériques - Typologie.

From the typology of digital tools in the field of CEHL to operational capability for inclusion

Summary: In the field of Computing Environments for Human Learning (CEHL), many digital resources are now available to teachers to facilitate the transmission of knowledge in general and to meet in particular the needs of pupils with disabilities or learning difficulties. The purpose of this research is to examine the operational capability for inclusion of these resources, that is to say to what extent these tools can help to contribute to universal educational accessibility. For this purpose, the methodology consisted in constructing a model of typological analysis in the form of a heuristic schema intended to map, by using a marker constituted by the crossing of two axes, that of didactisation and that of compensation/accessibility, the conceptual space for the elaboration and development of these tools. Their aim may be either specific or generic; focusing on the provision of deficiencies or the optimization of learning for all. The localization on the heuristic map of some digital tools with regard to their stakes and their internal logics makes it possible to make emerge the dynamic axis for measuring their inclusive operational capability for inclusion, transversal to the space structured by the two preceding ones.

Keywords: Accessibility - Capability for inclusion - CEHL - Compensation - Didactisation - Digital resources Disability - Typology. 
E service public d'éducation " veille à l'inclusion scolaire de tous les enfants, sans aucune distinction », lit-on à l'article 2 de la loi 2013-595 du 8 juillet 2013. C'est donc une obligation d'accessibilité du système scolaire qui se trouve ainsi créée. II s'agit de refonder l'École de la République afin de la rendre capable de prendre en compte toute la diversité des élèves et de permettre à chacun d'atteindre les objectifs scolaires visés pour tous.

Concrètement, un tel objectif de mise en accessibilité des environnements éducatifs, dans la perspective d'une éducation inclusive, signifie que l'on s'efforce moins de dégager les besoins éducatifs de l'apprenant dans son environnement que de dégager les besoins d'aménagement de cet environnement, pour le rendre exempt des obstacles et des barrières non seulement physiques, mais aussi didactiques, pédagogiques et linguistiques qui peuvent le rendre handicapant ou invalidant (Benoit, 2014a, p. 197; Ebersold, 2013b, p. 25; Fougeyrollas, 2009, 2010). Cette évolution est conforme au principe de la " conception universelle " (universal design) affirmé par l'article 2 la Convention relative aux droits des personnes handicapées (CRDPH, ONU, 2006), adoptée par la France en 2010, selon lequel la condition première de I'inclusion sociale apparaît comme celle de l'accessibilité de l'environnement, même si elle " n'exclut pas " (ibid.), dans un second temps, les réponses compensatrices individuelles.

D'un autre côté, les politiques de développement du numérique éducatif et de la pédagogie numérique, aussi bien dans l'enseignement scolaire ${ }^{1}$ que dans l'enseignement supérieur ${ }^{2}$, offrent une remarquable opportunité de mettre les usages du numérique au service d'une "école inclusive pour les élèves en situation de handicap " (Cnesco, 2016) et de les faire contribuer au renversement du processus de normalisation consistant à passer du " modèle d'intégration réadaptatif, essentiellement fondé sur la normalisation des individus" au modèle "d'accessibilisation des milieux de vie tels que l'école, l'entreprise, la cité » (Sanchez, 2000, p. 310).

L'appel à projets e-FRAN « espaces de formation, de recherche et d'animation numériques ", lancé par Najat Vallaud-Belkacem, ministre de l'Éducation nationale, de l'Enseignement supérieur et de la Recherche, et Axelle Lemaire, secrétaire d'État chargée du Numérique, le 8 octobre 2015, de même que le plan numérique annoncé par le Président de la République qui poursuit son déploiement en 2016, 2017 et 2018, sont des signes clairs du volontarisme de l'action politique dans ce domaine. L'objet de cet article est, tout d'abord, de présenter un modèle d'analyse typologique des outils numériques dans le champ des EIAH. Ce modèle prend la forme d'un schéma heuristique destiné à cartographier, notamment sous le prisme de l'accessibilité, l'espace conceptuel d'élaboration et de développement de ces outils (Benoit, 2013). Dans un second temps, il s'agira d'interroger la validité de ce modèle en le confrontant à quelques outils numériques pour l'éducation, puis, dans un troisième temps, de montrer comment cette démarche conduit à l'émergence du concept d'opérationnalité inclusive, et en quoi celui-ci constitue la dimension dynamique de cette cartographie, c'est-à-dire, comme on le verra, celle de l'accessibilisation.

1. Division du numérique éducatif au ministère de l'Éducation nationale (DNE).

2. Mission de la pédagogie et du numérique (MIPNES) au ministère de l'Enseignement supérieur. 
Dans le contexte actuel de l'omniprésence du numérique, cette recherche se développe à l'intersection de trois champs théoriques. Elle se réfère, d'une part, à l'analyse typologique des Environnements informatiques pour l'apprentissage humain (EIAH), qui a fait l'objet de beaucoup de travaux parmi lesquels nous retenons ceux de De Vries, (2001), Puimatto (2004), Bibeau, (2005), Baron et Harrari (2006). Elle s'appuie, d'autre part, sur le paradigme de l'accessibilité, qui a été documenté par de nombreux chercheurs (Fougeyrollas, 2009, 2010; Gardou, 2012 ; Larrouy, 2011; Sanchez, 2000; Ebersold, 2009, 2013, 2017; Benoit, 2007, 2008, 2009, 2012, 2014 ; Plaisance, 2013). Est convoquée enfin l'approche sociologique et ethnologique de l'outil et du geste technique, dans laquelle s'inscrit l'étude du " geste d'enseignement », particulièrement Iorsqu'il vise l'aide pédagogique, effectuée par Dunand et Feuilladieu (2014, p. 114). La notion de "degré de généricité-spécificité " du geste d'aide qu'introduisent ces auteurs dans leur analyse (2014, p. 117) articule compensation et accessibilité et constitue à ce titre l'une des composantes du concept d'opérationnalité inclusive.

\section{LE CONTEXTE: OMNIPRÉSENCE DU NUMÉRIQUE ET LIMITES}

À partir d'un Dossier d'actualité réalisé en 2012 par Rémi Thibert dans le cadre de I'IFÉ, on peut dégager quelques éléments de contexte éclairants sur le développement des relations entre pédagogie, numérique et apprentissages.

Il apparaît tout d'abord qu'aujourd'hui, à tous égards, sont dominantes les représentations selon lesquelles nous vivons une révolution d'aussi grande envergure que celle de I'invention de l'imprimerie aux XV et XVI ${ }^{e}$ siècles, comme l'affirme Michel Serres dans son ouvrage, Petite Poucette, publié en 2012. Au regard de l'intérêt réel ou supposé du numérique, les États, les collectivités, les institutions, ont massivement investi et continuent de le faire dans des équipements ou des ressources numériques. Malgré les enquêtes, recherches et méta-analyses qui ont souvent conclu à un impact modéré, voire inexistant sur les résultats des élèves, les technologies numériques suscitent beaucoup d'espoir et les financements pour les généraliser restent toujours très importants. Les décideurs des politiques publiques partent en effet du postulat que la technologie peut améliorer la qualité de l'éducation en rendant l'enseignement-apprentissage plus efficace et en favorisant du même coup un changement radical des pratiques éducatives (Thibert, 2012, p. 2).

Or, pour Thomas Russel, les méta-analyses sont convergentes et ne parviennent pas à établir de façon catégorique l'efficacité des TIC sur les apprentissages. C'est le phénomène NSD qu'il décrit dans son ouvrage The No Significant Difference Phenomenon (2001). D'après la recension de I'IFÉ, la plupart des analyses qui ont suivi valident cette thèse de l'absence de différence significative.

Mais pour Kirsch (2008), comme pour le think tank américain Educause, la question de l'efficacité est mal posée, car il est impossible d'isoler une variable en laissant de côté toutes les autres. L'apprentissage est la résultante de plusieurs facteurs: motivation, métacognition, processus interactifs, conflits socio-cognitifs, transfert des apprentissages dans d'autres contextes. Dans la même perspective l'étude de Barrette montre que "l'intégration des TIC peut être efficace quand elle soutient d'une manière adaptée les approches pédagogiques appropriées aux objectifs des programmes d'études » (2009, p. 19). En fait plusieurs études mettent en avant 
le rôle déterminant de l'enseignant et soutiennent que la question véritablement déterminante en matière d'efficacité est celle du contexte pédagogique. Dès lors, il ne s'agit pas tant de savoir (via l'Evidence-based education) s'il faut utiliser les TIC pour transmettre des contenus de connaissances, mais d'identifier les solutions techniques susceptibles de soutenir les élèves pour leur permettre d'atteindre un objectif d'apprentissage donné (Tamim, 2011 ; Duttta et Bilbao-Osorio, 2012, cités par Thibert, 2012).

En France, comme dans de nombreux pays, les équipements d'établissements se sont nettement améliorés, mais les difficultés résident dans le manque d'encadrement et de logiciels d'apprentissage appropriés (Eurydice, 2011). On observe que seuls $19 \%$ des enseignants font un usage pédagogique des TIC dans la classe, alors même qu'ils les utilisent pour préparer leurs cours (DEPP, 2010). II faudrait donc passer d'une logique d'équipement à une logique d'usage pédagogique des ressources numériques (plan DUNE, 2010). À cet égard, on aurait pu penser que la généralisation en cours des Environnements numériques de travail (ENT) contribuerait à cette évolution, mais ils sont plus souvent utilisés pour la gestion administrative des établissements scolaires qu'ils ne sont sources d'innovation pédagogique.

\section{CHAMPS THÉORIQUES ET LOGIQUES INTERNES DES OUTILS NUMÉRIQUES}

\section{Analyse typologique des artefacts informatisés en éducation}

La définition donnée par Gérard Puimatto ${ }^{3}$ (2004) des ressources multimédias ou numériques en éducation permet de prendre conscience du très large spectre dans lequel elles s'inscrivent: "... les "ressources multimédias pour l'éducation" constituent l'ensemble des informations, documents, logiciels, programmes, banques de données, etc. qui permettent de véhiculer, de transmettre ou d'appréhender des concepts et contenus d'enseignement. Le terme ressource est plus générique que celui de document, même dans l'acception multimédia du mot".

Partant de cette définition, Georges-Louis Baron et Michelle Harrari (2006, p. 38) signalent différents travaux entrepris pour établir des typologies d'artefacts informatisés utilisés en éducation:

- La classification des Environnements informatisés pour l'apprentissage humain (EIAH) proposée en 2001 par Erica de Vries (2001) qui se fonde sur l'intention qui a présidé à leur conception;

- La proposition de taxonomie des TIC à l'école de Robert Bibeau (2005), qui se donne pour but de "décrire et cataloguer ces ressources, particulièrement celles sur support numérique, selon les normes internationales de façon à en assurer le stockage, la portabilité et l'interopérabilité " (cité par Baron et Harrari, 2006, p. 38). Si Baron et Harrari assurent ne pas souhaiter ajouter aux précédentes une nouvelle typologie des ressources multimédias, ils n'en proposent pas moins un analyseur

3. Soutenue en juin 2006 à l'université de Paris 13 et effectuée sous la double direction d'Éric Bruillard et de Pierre Moeglin, la thèse de Gérard Puimatto est disponible en ligne sur le site Internet de I'INRP: http://www.inrp.fr/vst/Recherches/DetailThese.php?these $=670$ 
efficace de ces technologies, qu'ils appellent le "degré de didactisation ", et qui pourrait tout à fait fonctionner comme un critère de classement. II s'agit en fait de caractériser la plus ou moins grande intensité de la prescription pédagogique, ou si l'on veut, d'encadrement et de surveillance - au sens que prête à ce mot Michel Foucault (1975, p. 210) - de I'utilisation que fait l'apprenant de la ressource.

L'intérêt d'une telle caractérisation serait de ne pas enfermer les objets concernés dans des catégories réductrices, mais au contraire de les situer sur un axe, celui du plus ou moins grand degré de didactisation. Ainsi les auteurs sont-ils conduits à dégager trois ensembles, dont les contours n'auront pas vocation à être précisés avec exactitude, puisque le positionnement des différents objets sera établi de manière relative les uns par rapport aux autres.

Ils distinguent ainsi trois groupes de ressources numériques:

1. Les différents artefacts susceptibles d'être mis au service d'un projet, d'un objectif d'apprentissage, c'est-à-dire disponibles et potentiellement mobilisables pour l'éducation, mais ne proposant pas un cheminement pédagogiquement prédéterminé (Baron et Harrari, 2006, p. 38). II s'agit le plus souvent d'applications généralistes, qui n'ont pas été conçues spécifiquement dans un but éducatif, mais qui peuvent y contribuer, en s'inscrivant dans le cadre d'une démarche ou d'une activité pédagogique particulière. Ces activités peuvent avoir pour objet la recherche de documents, la rédaction d'un devoir, la préparation et l'illustration d'un exposé, d'un dossier, la réalisation d'un contrôle de connaissances. La plupart des logiciels de traitement de texte, avec leurs différentes fonctions, et des ressources en ligne (sites et moteurs de recherche, sites de type Wiki) peuvent être rangés dans cet ensemble, auquel on peut ajouter la téléphonie et la vidéo.

2. À l'opposé, figurent les ressources correspondant à proprement parler à des Environnements informatisés pour l'apprentissage humain (EIAH). Ces applications ont en commun une certaine rigidité, car les parcours des usagers y sont étroitement dirigés et surveillés et obéissent à une programmation didactique très stricte: un learning design ou design d'apprentissage. La part de liberté et d'initiative est par conséquent plutôt réduite. Ces outils, qui se sont aujourd'hui considérablement développés dans le secteur marchand de l'accompagnement scolaire, sont plutôt prescrits par les adultes, notamment les parents, que choisis par les utilisateurs: à ce titre ils ne correspondent qu'indirectement à I'idée d'une ressource.

3. À une place intermédiaire, se trouvent des instruments informatiques plus ou moins didactisés, utilisables dans un certain nombre de disciplines, par exemple sur demande des professeurs, comme des logiciels de dessin, de construction géométrique, de création et d'édition de tableaux de mesure et de graphiques. 
La dimension de la didactisation, correspondant aux processus d'apprentissage libres ou prescrits, constitue le premier axe de la construction du modèle typologique (figure 1).

Figure 1 : Axe 1 : degré de programmation didactique

\section{AXE DE LA DIDACTISATION}

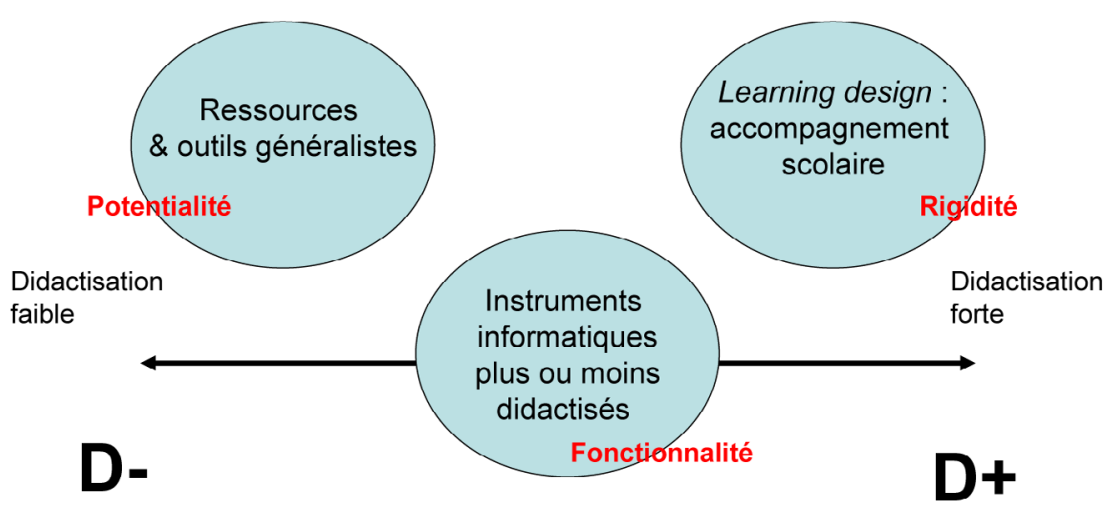

RESSOURCES

NUMERIQUES

\section{Numérique et handicap au regard du paradigme de l'accessibilité}

Au moment où se multiplient, en direction de toutes les filières de l'enseignement scolaire, les actions d'appui (sensibilisation, formation, accompagnement) à l'accueil et à la scolarisation de tous élèves dans leur diversité, et notamment ceux dont la situation de handicap ou de difficulté est reconnue, la question de l'accessibilité des parcours scolaires se présente tout autant comme une urgence politique que comme une obligation éthique.

La question de l'accessibilité de tous les supports pédagogiques utilisés se pose avec d'autant plus d'acuité que les enseignants ont besoin de s'appuyer sur des démarches et des ressources renouvelées pour construire et opérationnaliser de nouvelles compétences professionnelles face à la diversité des besoins auxquels ils sont désormais appelés à répondre ${ }^{4}$. La dynamique de numérisation dans laquelle se trouve engagée l'édition en général a ouvert la voie à une nouvelle donne de l'accessibilité de ces supports: qu'il s'agisse des adaptations relatives à la déficience visuelle (braille, dessin en relief), aux déficiences motrices (outils de traçage) ou aux troubles du langage (logiciels de mise en page ou de synthèse et de transcription vocales), aux troubles cognitifs ou à la surdité (Langue des signes française - LSF, Langue française parlée complétée - LfPC), des ressources numériques adaptatives en

4. Article 2 de loi 2013-595 du 8 juillet 2013, cité plus haut. 
ligne permettent déjà de répondre en partie aux besoins des élèves en situation de handicap.

Dans le domaine du handicap et de la difficulté scolaire, les ressources numériques se sont historiquement développées sous la forme d'aides techniques personnalisées en relation avec des déficits et/ou des troubles moteurs, cognitifs ou sensoriels: faire plus, faire vite et faire mieux par les applications augmentatives; apprendre de façon différenciée, à son rythme et dans un rapport particulier avec l'enseignant par les activités de type tutoriel; organiser sa pensée, développer ses stratégies et acquérir non plus seulement des connaissances mais des procédures et des méthodes par des activités de type procédural; consulter des ressources et échanger dans le cadre d'applications communicationnelles. C'est dans cette logique que le ministère de l'Éducation nationale installe, en novembre 2007, I'Orna (Observatoire des ressources numériques adaptées) sous l'égide de I'INS HEA.

L'efficacité de l'aide apportée par ces applications reste néanmoins très fortement liée au type d'approche de la situation et de l'activité d'apprentissage. À l'école, au collège ou au lycée, l'élève, dont la situation de handicap est en interaction dynamique ${ }^{5}$ avec un type de trouble, moteur, sensoriel ou cognitif, est confronté à une multitude de tâches dans lesquelles il peut rencontrer d'importantes difficultés. L'écoute de l'enseignant, la compréhension et la mémorisation d'une consigne, la lecture de documents imprimés et la production d'une réponse écrite, la résolution d'un problème mathématique, le calcul numérique, le traçage géométrique, la consultation et la sélection d'informations, la communication et l'échange sont autant de tâches scolaires d'apparence banale qui sont souvent associées dans une activité. Elles renvoient à une imbrication d'opérations mentales, mobilisant aussi bien les fonctions exécutives ${ }^{6}$ de l'élève que la perception, la motricité ou même les émotions, et qui entraînent une dépense importante d'énergie cognitive. L'existence pour tel élève d'une limitation particulière liée ou non à un trouble sensoriel, moteur, ou cognitif peut entraîner pour l'une ou l'autre de ces activités un coût cognitif véritablement exorbitant, au point d'épuiser complètement ses ressources attentionnelles et de l'empêcher d'aller au terme de l'activité dans son ensemble (Benoit et Sagot, 2008). Mais la réponse adaptée à ce besoin ne se décline pas seulement sur le plan de l'accessibilité individuelle, elle renvoie dialectiquement à la dimension collective de l'action pédagogique, à travers l'analyse de la situation didactique et des contraintes et exigences plus ou moins visibles qu'elle impose à tous les apprenants. C'est à cette condition que les contextes d'apprentissage peuvent être améliorés pour tous et former des environnements universellement facilitants (Benoit, 2012b, p. 97).

5. "L'état de fonctionnement et de handicap d'une personne est le résultat de l'interaction dynamique entre son problème de santé (maladies, troubles, lésions, traumatismes, etc.) et les facteurs contextuels. " (CIF, p. 8 http://apps.who.int/iris/bitstream/10665/42418/1/9242545422_fre.pdf).

6. "Selon Seron, van der Linden, et Andres (1999), les fonctions exécutives (FE) consistent en un "ensemble de processus dont la fonction principale est de faciliter l'adaptation du sujet à des situations nouvelles, notamment lorsque les routines d'actions [...] ne peuvent suffire." " Cité par Monette et Bigras (2008, p. 323) qui ajoutent que " cette définition, bien que surinclusive selon plusieurs, obtient un certain consensus dans la communauté des chercheurs". 
C'est pourquoi on ne peut affirmer que le développement du numérique serait naturellement générateur d'environnements accessibles, attaché qu'il est traditionnellement à la suppléance, la rééducation ou la réparation de facultés cognitives, sensorielles ou mentales déficientes. En répondant souvent terme à terme à des besoins individuels qui peuvent être définis comme "I'envers positif de la répercussion du trouble » (Benoit, 2012a, p. 76; 2014a, p. 197), les TIC renvoient à une logique indexée à la nosologie des troubles et pathologies.

Or l'ajustement des supports d'apprentissage et la production d'outils numériques n'a de sens que dans le cadre d'une démarche d'accessibilité pédagogique globale visant à rendre capacitante pour tous la situation d'enseignement-apprentissage. II s'agit alors de questionner les normes admises afin de produire des scénarios pédagogiques où l'on s'efforce moins de compenser des manques que d'identifier et de lever les obstacles qui adhèrent à la situation d'apprentissage (Benoit, 2009, p. 33). II pourrait être contre-productif de se contenter d'attribuer des réponses compensatrices spécifiques à une organisation physiologique dite déficitaire, comme on apporterait la pièce manquante à un puzzle humain imparfait, qui, ainsi complété, deviendrait assimilable à un élève normalisé, en indifférence au contexte. La neutralisation de l'environnement et la focalisation sur les répercussions des troubles aurait pour effet, d'une part, d'exonérer l'enseignant ou l'éducateur de I'identification des éventuelles barrières présentes dans la situation d'apprentissage et, d'autre part, de faire peser un risque de stigmatisation et de marginalisation sur la personne concernée. De telles actions compensatrices correspondent à l'attribution administrative d'aides additionnelles destinées à pallier de manière ponctuelle les difficultés individuelles rencontrées par un élève considéré comme handicapé. En résumé, que peuvent apporter aux apprentissages ces aides techniques si elles ne se décentrent pas du déficit individuel pour s'inscrire dans la cohérence d'une posture pédagogique interactive, collaborative et inclusive?

L'inclusion scolaire est en effet inséparable d'une logique de refondation pédagogique et institutionnelle au sens ou Simona D'Alessio, s'appuyant sur les travaux d'Armstrong et Barton, la définit " comme un processus de transformation des systèmes éducatifs et des cultures qui permet à tous les élèves de participer entièrement et équitablement au processus d'apprentissage dans les classes ordinaires " (2009, p. 36). Elle ajoute que le développement de l'éducation inclusive entraîne une "déconstruction " des notions actuelles de scolarisation, d'apprentissage et d'éducation afin d'établir les pré-conditions de l'apprentissage de tous (ibid. p. 44).

À l'inverse de l'accessibilité qui implique une dialectique de l'individuel et de l'universel et qui engage vers la transformation des systèmes et des fonctionnements scolaires et sociaux, la compensation est une dimension essentiellement centrée sur l'individu et sur le traitement des répercussions des déficiences et des manques qui ont été diagnostiqués chez lui. Les procédures et les outils rééducatifs, qui isolent et ciblent une fonction pour tenter de la réparer par un protocole d'exercices appropriés, s'inscrivent dans ce cadre. Ils se focalisent sur la suppléance ou la restauration de facultés physiologiques déficientes et fonctionnent la plupart du temps comme des compensations normalisantes. 
Du point de vue juridique, la compensation est toujours individuelle. L'article L.146-8 du CASF (Code de l'action sociale et des familles) précise par exemple: "Une équipe pluridisciplinaire évalue les besoins de compensation de la personne handicapée et son incapacité permanente [...] et propose un plan personnalisé de compensation du handicap. "Elle est considérée du point de vue de la personne handicapée elle-même; elle regroupe l'ensemble des mesures prises en faveur d'une personne pour combler les déficits, rattraper les retards, c'est-à-dire en définitive pour réduire l'écart à la norme. Elle concerne toutes les dimensions de la vie sociale, professionnelle et scolaire, mais sur le plan individuel. Elle se présente comme une compilation, une combinatoire de mesures additionnelles spécifiques, en dehors et au-delà du droit commun.

Les dispositions sociales et scolaires prises au regard du handicap, comme d'ailleurs de la (grande) difficulté, peuvent donc s'inscrire sur un axe allant de la compensation - qui renvoie à une approche individuelle consistant à apporter des aides à l'élève en fonction de ses capacités/incapacités et déficiences - à l'accessibilité - qui renvoie à une approche collective et environnementale du contexte handicapant ou invalidant (Creai Rhône-Alpes, 2011). Dans cette perspective, il est possible de situer sur le curseur compensation-accessibilité les différents types de ressources numériques sollicitées en tant qu'aides techniques pour l'éducation des jeunes en situation de handicap ou de difficulté. La dimension de la compensation et de I'accessibilité, correspondant au rapport au contexte centré sur l'individu ou bien ouvert sur le collectif, constitue le deuxième axe de la construction du modèle typologique (figure 2).

Figure 2 : Axe 2 : collectif / individuel

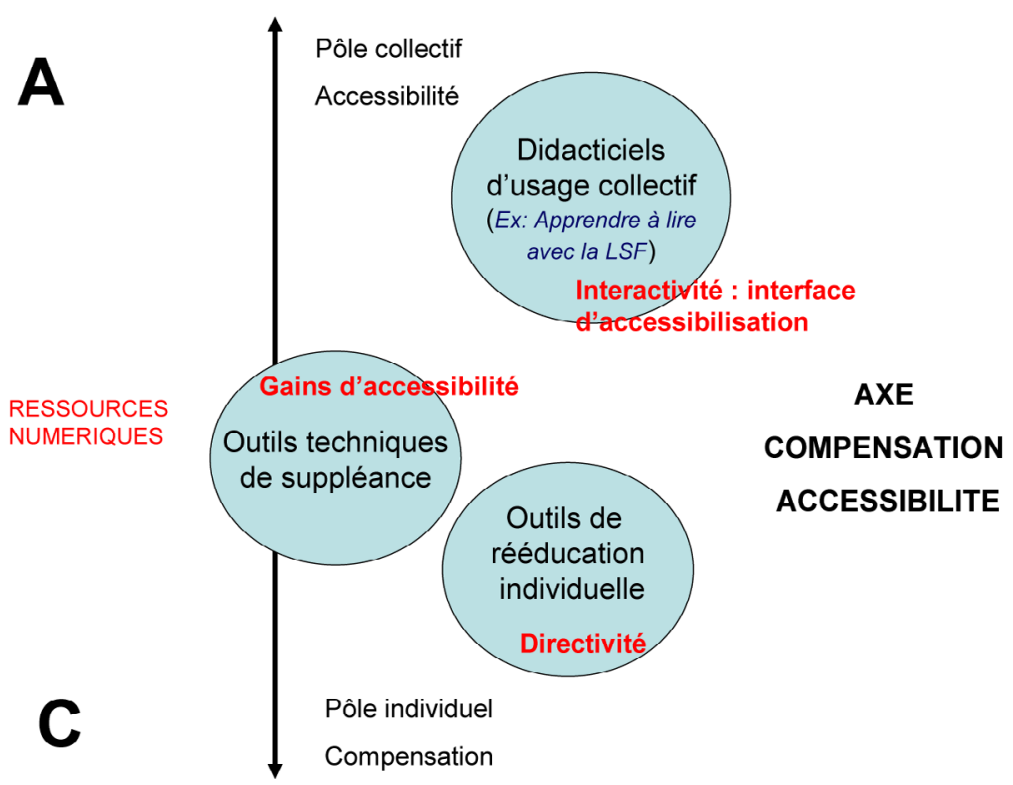




\section{Au croisement des axes de la didactisation et de l'accessibilité: un espace d'élaboration}

Ces différents outils peuvent également être positionnés sur l'axe du degré de didactisation, ce qui permet de combiner les deux analyseurs en croisant les axes et de définir le schéma ou la carte heuristique permettant de structurer l'espace conceptuel d'élaboration et de développement des ressources. Les outils de rééducation individuelle (par exemple, en orthophonie) sont des compensations fortement didactisées; les outils techniques de suppléance (par exemple, le preneur de notes électronique pour les aveugles ou aides au tracé géométrique pour les troubles moteurs) sont eux aussi des compensations, présentant un faible degré de didactisation, mais permettant des gains importants d'accessibilité, dès lors qu'ils sont utilisés en milieu scolaire ordinaire et qu'ils contribuent à l'inclusion. Les didacticiels du type J'apprends à lire avec la LSF 7, qui seront examinés plus loin, sont à la fois fortement didactisés et nettement orientés vers l'accessibilité. Les enjeux théoriques de l'élaboration des outils numériques dans le champ de l'accessibilité se situent donc au croisement des deux logiques. L'examen du point d'articulation de ces deux premiers questionnements, à l'intersection de l'axe de la didactisation et de l'axe de la compensation et de l'accessibilité, permet de cartographier les pistes et les impasses au regard de l'éducation inclusive.

L'intersection de l'axe de la didactisation et de l'axe de la compensation-accessibilité constitue la troisième étape de la construction du modèle typologique (figure 3 ).

Figure 3 : Croisement des axes 1 et 2

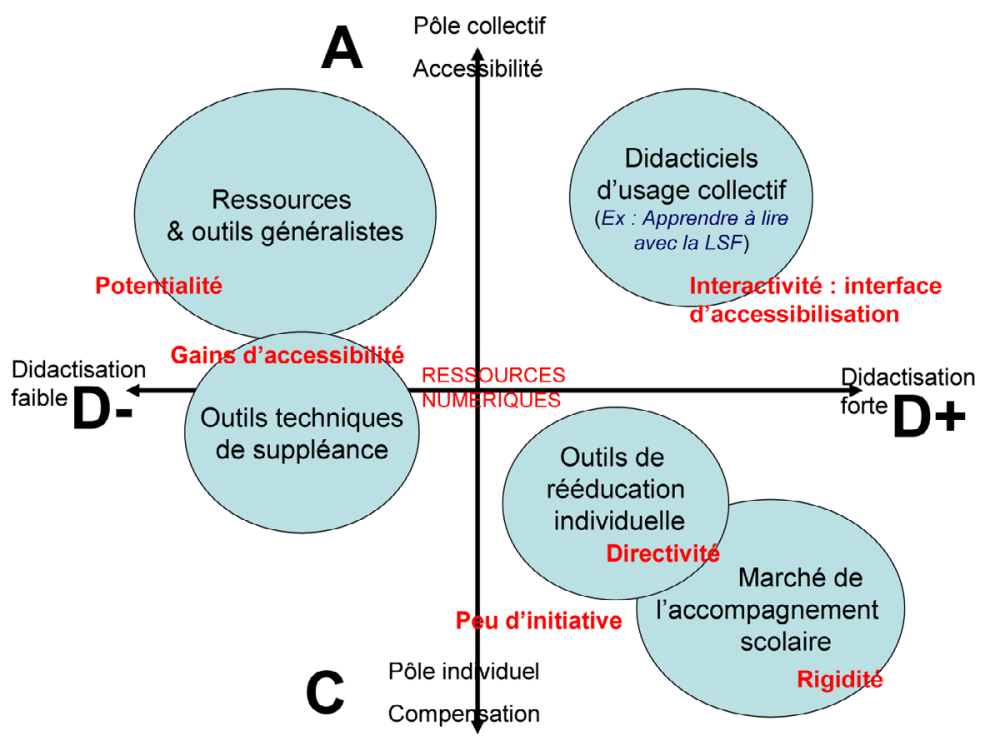

7. Voir exemple 3. 
À partir de la carte heuristique constituée par le croisement des deux axes de la didactisation et de la compensation-accessibilité, quatre zones de ressources sont définies: la "ZAD- " correspond aux outils génériques potentiellement générateurs d'accessibilité et faiblement didactisés; la " ZCD- ", à ceux qui, faiblement didactisés, relèvent de la compensation; la " ZCD+ " aux instruments compensatoires fortements didactisés et la "ZAD+ " aux applications qui, tout en étant fortement didactisées, contribuent néanmoins à l'accessibilité.

La zone la moins riche en outils, et qui ne correspond actuellement à aucun marché, est la "ZAD+ » : tout se passe comme si l'augmentation du degré de didactisation entraînait une focalisation sur les manques et déficits individuels, que ceux-ci se situent ou non dans le champ du handicap. La zone la plus riche est la "ZAD- », puisqu'elle englobe l'ensemble des ressources numériques potentiellement disponibles pour l'éducation de tous. Un grand nombre d'entre elles offrent d'ailleurs un potentiel d'outils de suppléance plus efficaces et moins stigmatisants ( ZCD-») que des matériels très spécialisés, très puissants et souvent très chers ( ZCD+ "), qui peuvent ne servir à rien et même parfois alourdir la scolarisation s'ils ne répondent pas à un vrai besoin qui poussera l'élève à apprendre à s'en servir (Benoit et Sagot, p. 23) . Comme on le verra plus loin (exemple 3), le domaine de la surdité est à ce titre emblématique, en ce que la centration sur le traitement compensatoire du déficit auditif ( $Z C D+»)$, la recherche et la mise en œuvre de solutions à l'écart à la norme, conduisent à une impasse éducative en termes d'accessibilité. L'effectivité de l'accessibilisation, c'est-à-dire de l'opérationnalité inclusive de l'outil, est dans ce cas en effet conditionnée à la prise en compte du signeur comme locuteur de plein droit et à la reconnaissance de la modalité gestuelle de communication comme langue à part entière. II est donc cohérent que des didacticiels comme J'apprends à lire avec la $L S F$ se trouvent en "ZAD+ ", tandis que les outils de réparation orthophonique s'inscrivent en "ZCD+ ». Force est de constater que les ressources numériques s'appliquent le plus souvent à traiter la différence individuelle en s'efforçant de lui apporter la résolution de l'écart à la norme, tandis qu'elle aurait besoin de la reconnaissance de leur besoin en situation.

\section{L'outil, le geste technique et le degré de " généricité-spécificité "}

Comme le montrent Dunand et Feuilladieu dans une étude (2014, p. 115) portant sur les aides pédagogiques aux élèves dyslexiques, la question des aides spécifiques apportées à ces élèves, pensées par les enseignants comme rendant accessibles les apprentissages (notamment dans le domaine de la maîtrise de la langue), intéresse la réflexion sur les gestes d'enseignement génériques, c'est-à-dire ceux qu'ils utilisent pour les autres élèves de la classe et qu'ils remanient ou non à des fins d'individualisation. De nombreuses études ont en effet montré que les pratiques pédagogiques font elles-mêmes parfois obstacle à l'entrée dans les apprentissages d'élèves en difficulté, sans dysfonctionnement diagnostiqué, à travers la manière dont les enseignants élaborent les exercices, lorsque les objectifs de travail ou les savoirs en jeu dans les tâches scolaires demandées sont opaques ou trop implicites pour être perçus et investis dans l'activité par l'élève (Bonnéry, 2007; Lahire, 2008). 
Les gestes d'aide, appréhendés à travers leur degré de généricité-spécificité, matérialisent I'hexis corporelle des enseignants, cette disposition permanente incorporée du métier selon Bourdieu (2000), à la fois en tant que capacités de faire - pratiques routinisées permettant d'agir - et capacités à faire - aptitude à développer et créer des solutions pratiques. La combinaison de gestes à polarité principale générique (accessibilité) et de gestes à polarité principale spécifique (compensation), concrétise cette double acception de disposition. Ces polarités décrivent plus que les gestes techniques d'aide à l'étude. Elles témoignent de l'articulation des routines et des usages innovants que permettent ces routines.

Former les enseignants aux gestes techniques sans les former aux démarches dans lesquelles ils s'insèrent et aux postures auxquelles ils correspondent, dissocie ces gestes de la pratique du métier et les désincarne pour les réduire à une application non signifiante. Or, c'est bien l'imbrication des gestes au sein d'une posture qui rend la pratique du métier opérante. Par exemple en ce qui concerne l'enquête présentée sur les gestes d'aide à l'étude envers les élèves dyslexiques, les résultats montrent la pertinence de contenus de formation qui poseraient ces gestes comme une manière possible d'enseigner de façon ordinaire, et non comme une manière spécifique de s'adresser à certains élèves (Feuilladieu, Dunand, 2015).

Séparer l'outil et ses effets des usages techniques, comme cela peut être le cas lorsqu'on considère l'outil tel qu'il se présente dans sa fonction, amène à confondre outil et action technique. Or, I'outil n'est pas la technique, comme l'ont très bien montré les ethnologues: "C'est à ce niveau [geste élémentaire technique] que s'exprime souvent le style technique, là aussi qu'est le mieux mise en évidence la relation entre geste et outil dans l'action technique. [...] L'action technique ne se confond pas avec l'outil. Quelle que soit la valeur de celui-ci comme témoin, ce n'est pas lui [l'outil] mais le geste qui constitue l'atome technique: non pas le fléau, ou la navette, mais battre le fléau, lancer la navette... geste qui intègre l'outil, qui le rend efficace et lui emprunte la forme spécialisée de son action sur la matière. " (Cresswell, p. 53).

\section{LA TYPOLOGIE À L'ÉPREUVE DE QUELQUES EXEMPLES D'OUTILS NUMÉRIQUES}

\section{Exemple 1: Production d'un texte documentaire à partir de la consultation d'une base de données textuelle (Marin, 2008)}

Dans un article publié en 2008 dans La nouvelle revue de l'adaptation et de la scolarisation, Brigitte Marin décrit une recherche conduite auprès des élèves de deux classes de $4^{\mathrm{e}}$ de Segpa et de deux classes de $4^{\mathrm{e}}$ ordinaires dans un collège situé dans une zone socio-économiquement défavorisée de la banlieue parisienne. L'activité proposée aux élèves est de produire un texte documentaire scientifique et l'objet de la recherche consiste à étudier l'incidence d'un environnement numérique de travail sur la qualité des résultats obtenus. Chacun des deux groupes d'élèves de $4^{\mathrm{e}}$ ordinaire (46) et de $4^{\mathrm{e}}$ de Segpa (30) est réparti en deux sous-groupes de nombre équivalent travaillant I'un devant écran d'ordinateur et l'autre sur support papier. 
Après avoir produit un premier jet sur la question environnementale de la pollution des eaux douces, les élèves sont invités à prendre connaissance d'une banque de dix textes documentaires scientifiques portant sur ce sujet et à prendre des notes en s'aidant d'une liste de dix mots-clés. Ils ont pour consigne de lire avec une attention particulière les informations présentes dans les phrases contenant les mots-clés. Les groupes travaillant sur support numérique se reportent aux mots-clés à l'aide de la recherche automatisée (Ctrl F) et rassemblent les informations sélectionnées en utilisant la fonction copier/coller (Ctrl C/Ctrl V), tandis que ceux qui travaillent sur papier doivent repérer les mots-clés lors de la lecture du texte et recopier sur une feuille les informations qu'ils jugent importantes.

Au moment de la production du deuxième jet, les élèves disposent de leur texte initial corrigé par les enseignants et des notes qu'ils ont prises lors de la séance de lecture. Ce texte est fourni sous forme de fichier électronique aux élèves qui travaillent sur ordinateur et sous format papier aux autres. Les élèves ont pour consigne d'améliorer leur texte en l'enrichissant grâce aux nouvelles connaissances acquises à partir de la lecture des textes de la base de données.

Les analyses statistiques réalisées en vue de rendre compte des effets de la consultation des textes documentaires sur la qualité de la production croisent trois facteurs: le facteur groupe (classe de $4^{\mathrm{e}}$ Segpa vs classe de $4^{\mathrm{e}}$ ordinaire), le facteur modalité de travail (support numérique vs support papier) et le facteur de pertinence des ajouts opérés par les élèves lors de la réécriture de leur premier jet. Elles font notamment apparaître que dans la modalité de travail sur ordinateur, le nombre d'informations très pertinentes ajoutées par les élèves du groupe de $4^{\mathrm{e}}$ Segpa est presque équivalent à celui des élèves du groupe de $4^{\mathrm{e}}$ ordinaires. L'écart s'est réduit par rapport aux fortes différences observées dans la modalité de travail sur support papier, dans laquelle les résultats des élèves de $4^{\text {e }}$ Segpa sont 2 fois plus faibles que ceux des élèves des élèves de $4^{\mathrm{e}}$ ordinaire. L'auteur observe que la mise à disposition de ressources numérisées a produit deux effets: "favoriser le traitement et l'appropriation des informations fournies ; réguler les écarts entre élèves de classes différentes".

La recherche automatisée des mots-clés a permis une lecture sélective évitant le balayage intégral des textes à la recherche des noyaux informationnels, rendus ainsi accessibles, sans dépense excessive d'énergie cognitive liée aux processus de bas niveau. La capacité attentionnelle ainsi libérée a pu être investie dans l'activation de compétences de haut niveau, relatives à la construction de la cohérence de la signification du texte lu et à la verbalisation de cette représentation mentale dans l'activité rédactionnelle.

\section{Analyse de l'exemple 1 sous le prisme du modèle typologique}

Les fonctions recherche automatisée et copier coller du logiciel de traitement de texte Word font partie des applications généralistes, qui n'ont pas bien évidemment été conçues spécifiquement dans un but éducatif et encore moins dans un but d'éducation inclusive. Du point de vue de la didactisation, elles s'inscrivent donc dans l'espace "ZD- " correspondant aux ressources peu ou pas didactisées.

Sur l'axe vertical, en tant que potentiellement conçues pour être utilisées par tous, elles se situent plutôt du côté du pôle collectif que du côté du pôle individuel. C'est 
pourquoi la première localisation semble s'inscrire dans la "ZAD- » de l'espace conceptuel dessiné par le modèle typologique. Cette localisation dans le champ de l'accessibilité de la carte heuristique se trouve confirmée par l'usage qui est fait de ces fonctions comme outil de suppléance des procédures de bas niveau (balayage du texte pour retrouver les mots-clés) pour tous les élèves. Celui-ci n'a pas eu seulement pour effet de réduire les écarts de performance entre des élèves réputés en grande difficulté scolaire et des élèves dits ordinaires, il a aussi considérablement accru le niveau de performance, en termes d'inférences, de hiérarchisation et de mise en cohérence des informations, des élèves ordinaires travaillant sur support numérique par rapport à ceux travaillant sur papier.

Le bénéfice capacitant partagé par tous de l'usage des fonctions numériques considérées conduit à repenser, au-delà de cet exemple, la relation entre compensation et accessibilité comme une opposition dialectique de l'individuel et de l'universel plutôt que comme une dichotomie, comme une synallagmatique évolutive plutôt que comme une antinomie définitive. Même une intervention compensatrice, instrumentée par des outils rééducatifs ou de suppléance des déficits, peut trouver place dans une logique d'accessibilité, à la condition de resituer la fonction cognitive concernée dans la situation d'enseignement où elle est sollicitée, au regard des enjeux de savoirs, des obstacles didactiques et du panel de compétences de bas niveau et de haut niveau à activer pour un apprentissage réussi. On voit bien qu'il ne s'agit pas alors de la simple transposition d'une adaptation ou d'une suppléance individuelle à une ou plusieurs autres personnes, mais de l'enchâssement des aménagements capacitants dans le sol didactique de l'action d'enseigner.

Au regard de l'axe horizontal, on peut observer que le fait que les chercheurs aient mis des fonctions initialement non didactisées au service d'une démarche très structurée pour faciliter la production d'un texte documentaire scientifique en allégeant la charge mentale liée aux processus cognitifs de bas niveau, peut être analysé comme un processus de didactisation secondaire, assurant l'efficacité d'acquisition et la restitution des connaissances, qui fait migrer l'outil ainsi contexualisé de la "ZAD- " à la « ZAD+".

Ainsi voit-on se dessiner une dimension dynamique qui traverse l'espace conceptuel d'élaboration des outils numériques et qui peut faire migrer certains d'entre eux d'une zone à l'autre (ici de la "ZD- » vers la "ZD+ »). Cette migration est consécutive à la modalité d'opérationnalisation de l'outil dans le sens de l'éducation inclusive. Elle peut s'effectuer sur l'un et l'autre axe de la carte heuristique.

\section{Exemple 2: Rôle de la motricité manuelle dans l'apprentissage de l'écrit. Représentation cérébrale et mémorisation des lettres (Velay, 2005)}

Dans un rapport de fin de recherche intitulé De la plume au clavier: est-il encore utile d'enseigner l'écriture manuscrite? (Velay, 2003), dont certains éléments sont repris dans un article publié deux ans plus tard dans Cerveau et psycho (Velay et Longchamp, 2005), les auteurs analysent le rôle de la motricité manuelle en jeu dans l'apprentissage de l'écriture sur la représentation cérébrale des caractères alphabétiques et par suite sur les aptitudes à lire. 
Les deux premières études permettent de mettre en évidence l'existence chez les adultes d'une composante sensorimotrice dans la représentation cérébrale des caractères alphabétiques. La mémoire motrice, plus stable dans le temps, vient en appui à la mémoire visuelle. L'existence de la composante sensorimotrice est confirmée par l'observation que "les zones du cerveau qui s'activent lorsqu'on reconnaît les lettres sont des zones qui participent normalement à l'écriture ". Aussi les chercheurs concluent-ils que les lettres seraient représentées dans le cerveau de façon "plurimodale ", c'est-à-dire qu'on les perçoit non seulement par la vue, mais aussi par la simulation mentale (inconsciente) des mouvements exécutés en écrivant. C'est donc un réseau neuronal étendu qui gouvernerait les processus conjoints du lire-écrire (Velay et Longchamp, p. 5-6).

La troisième étude de la recherche consistait à comparer, chez des enfants de maternelle, l'apprentissage traditionnel de la lecture/écriture et l'apprentissage avec un clavier. Les résultats préliminaires suggèrent que, pour les plus âgés, écrire les lettres à la main permet de mieux les mémoriser, mais que, chez les plus jeunes, c'est l'écriture au clavier qui donne les meilleurs résultats. II apparaît donc qu'il serait contre-productif d'entreprendre trop précocement l'apprentissage de l'écriture manuscrite avec des enfants (en dessous de 50 mois) qui ne contrôlent pas suffisamment leur motricité fine. En revanche, priver les enfants plus âgés (au-dessus de 50 mois) de cet apprentissage risquerait de compromettre le bon développement de leur compétence lexique. En effet, lorsqu'on apprend à lire en même temps qu'on s'initie à l'écriture manuscrite avec un outil scripteur, la vue des lettres est mentalement associée aux gestes accomplis par la main pour les former. La mémoire du geste consolide la représentation cérébrale et contribue ainsi à la mémorisation des lettres.

En résumé, chez les plus âgés (4-5 ans), l'écriture manuscrite augmente les performances de reconnaissance et de mémorisation des lettres; chez les plus jeunes (3 ans), on ne remarque pas d'écart dans ce domaine entre écriture manuscrite et écriture numérique.

\section{Analyse de l'exemple 2 sous le prisme du modèle typologique}

La fonction clavier d'un logiciel de traitement de texte relève des applications généralistes, sans objectif initial éducatif ni inclusif. Du point de vue de la didactisation, elle s'inscrit donc dans l'espace " ZD- » correspondant aux ressources peu ou pas didactisées.

Utilisée pour permettre, en classe maternelle ou élémentaire, à un enfant présentant un trouble dyspraxique d'entrer dans l'apprentissage de la lecture-écriture au même rythme que ses pairs, cette fonction constitue une adaptation compensatrice individuelle de la "ZCD- ", susceptible de migrer vers la "ZAD- » en tant qu'elle fonctionne comme un outil technique de suppléance du geste grapho-moteur offrant des gains significatifs d'accessibilité à un élève dyspraxique, pour lequel les tâches de motricité fine entraînent un coût cognitif exorbitant.

Mais cette fonction clavier peut aussi être proposée à tous les élèves en classe de petite section de maternelle (3-4 ans) et à ce titre se trouver enchâssée dans le sol didactique. Elle évolue alors vers la " $Z A+D-$ », et vers la " $Z A+D+$ " si elle est 
intégrée dans un scénario didactique structuré. Cette migration vers la "ZA+D+» renvoie à la contextualisation didactique qui permet d'assurer l'efficacité d'acquisition et la restitution des connaissances.

Comme dans le cas de l'exemple 1, c'est la modalité d'opérationnalisation de l'outil ou de la fonction numérique utilisée qui le fait migrer, sur la carte heuristique, soit, sur l'axe vertical, de la zone " $C$ » vers le bas de la zone " $A$ " (compensation individuelle avec gains d'accessibilité), voire vers le haut de ce même axe $A$ désigné par " $A+$

" (par enchâssement dans le sol didactique), soit, sur l'axe horizontal, de la zone « D- " vers la zone " D+ » (par intégration dans un scénario didactique structuré).

\section{Exemple 3: I'application " J'apprends à lire avec la LSF 1 et 2 "}

Les didacticiels J'apprends à lire avec la LSF 1 et $2^{8}$ sont des livres de lecture numériques conçus pour contribuer à l'accessibilité pédagogique de l'apprentissage de la lecture pour les élèves sourds, dans une logique inclusive. La langue des signes française, en tant que langue naturelle des enfants sourds signants, est pour les deux applications la langue de travail, dans laquelle sont proposées les consignes pédagogiques aux élèves, y compris dans le cadre du développement des compétences phonologiques et de la conscience métaphonologique.

Ainsi l'application prend-elle en compte les dimensions graphophonologique et syllabique de l'écrit, non pas à des fins de rééducation, mais pour ne pas couper artificiellement le lien entre oral et écrit. D'autre part, l'intégralité des propos signés est doublée en voix off et l'utilisateur a la possibilité de choisir un professeur sourd en LSF ou un professeur entendant qui s'exprime en français oral. Ainsi élèves et enseignants sourds et entendants peuvent travailler ensemble à partir du même support, sans entrave liée à leurs modes de communication respectifs et sans nécessité d'enseignement séparé pour les élèves sourds.

Dans I'opus 2, Monica Companys signe l'histoire intitulée Le ballon perché, publiée, comme celle du premier opus, Les Peurs de Petit-Jean, de manière classique aux éditions Bayard. De la lampe merveilleuse du texte écrit, effleurée d'un geste de la main par un enfant sourd, prénommé Aladin (ou Salim), jaillit le professeur narrateur signant, qui est la figure clé de cette génération de livres numériques au service de l'accessibilité pédagogique.

8. L'opus 1 est édité sous forme de DVD (disponible sur www.inshea.fr, boutique en ligne), l'opus 2 est accessible en streaming sur un site provisoire (http://agence-o.com/client//sf - identifiant " Salim » et mot de passe "Ballon »), avant d'être installé prochainement sur le site du CNDP Scérén. 


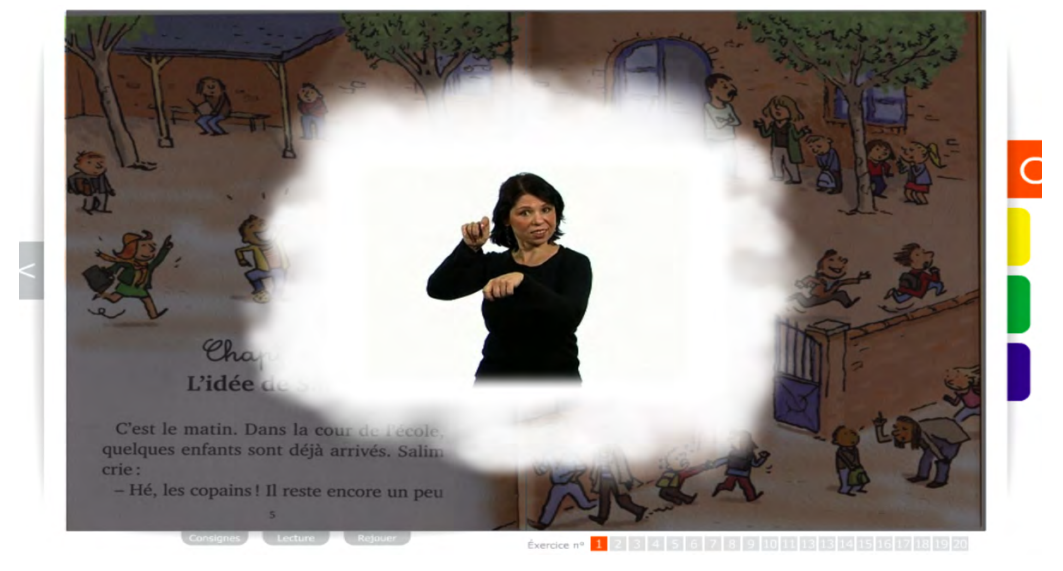

\section{Analyse de l'exemple 3 sous le prisme du modèle typologique}

Les applications numériques J'apprends à lire avec la LSF, au contraire des deux exemples précédents, sont des ressources très clairement positionnées dans la zone de haute didactisation (axe horizontal « $D+»$ ), avec un scénario didactique très structuré, et qui néanmoins contribuent fortement à l'accessibilité (axe vertical « $A+»)$. Avant toute opérationnalisation inclusive, ils prennent place dans la "ZAD+ ". On peut se demander pourquoi, dans ce cas, l'augmentation du degré de didactisation numérique n'a pas abouti à une focalisation sur les manques et déficits individuels. II faut sans doute en chercher l'explication dans la posture et l'intention de départ, qui visaient explicitement le renversement de cette tendance. II s'agissait en effet de la reconnaissance du sourd signeur (ou locuteur de la langue des signes), comme être de langage et de culture dans la modalité visuo-gestuelle, au rebours de son identification à une oreille déficiente, ce qui prémunissait efficacement le projet contre la directivité et la rigidité des outils de rééducation individuelle de la "ZCD+ ". Dans cette configuration, l'opérationnalité inclusive, qui s'est présentée dans les exemples 1 et 2 comme une dimension dynamique intervenant secondairement pour influer sur le positionnement des outils numériques sur la carte heuristique, a été effectivement intégrée dans la conception première de l'application de l'exemple 3, selon le principe de " conception universelle ", c'est-à-dire d'accessibilité universelle (universal design), tel qu'affirmé par l'article 2, cité plus haut, de la Convention relative aux droits des personnes handicapées (CRDPH, ONU, 2006).

\section{CONCLUSION}

Le numérique pensé pour l'accessibilité individuelle ne permet pas forcément I'accessibilisation, car son effectivité reste subordonnée au facteur d'opérationnalité inclusive. Ce facteur constitue l'axe dynamique susceptible de faire migrer les outils numériques considérés d'une zone à l'autre, et sur les deux axes de la carte heuristique. Le modèle typologique des EIAH présenté est ainsi traversé par l'axe 
moteur de l'opérationnalité inclusive, déterminant l'accessibilisation, qui renvoie à l'emploi qui est fait de la fonction ou de l'outil dans le cadre d'une démarche et d'une posture inclusives. Cet axe moteur permet d'infléchir par l'usage en contexte les logiques internes des ressources numériques. L'opérationnalité étant entendue comme ce qui permet d'agir (Le petit Robert), et l'épithète inclusive, comme ce qui concerne tout le monde, l'opérationnalité inclusive se définit comme une utilisation de l'outil numérique qui rend l'élève capable d'agir comme ses pairs dans la situation d'enseignement-apprentissage en le prémunissant contre le hors jeu didactique. L'opérationnalité inclusive n'est pas nécessairement liée à une didactisation préalable de l'outil, celle-ci pouvant être ou non intégrée à sa conception. La confrontation du modèle d'analyse typologique à différents artefacts informatisés pour l'éducation fait apparaître comme déterminante l'utilisation pédagogique et didactique de l'outil, à travers les gestes et les postures opérantes et signifiantes de cette utilisation. Les gestes, en tant qu' " habiletés acquises par apprentissage permettant la réalisation d'une tâche orientée vers un but spécifique " (Bril et Roux, 2002), prennent sens parce qu'imbriqués aux postures professionnelles, ces " manières d'aborder un problème posé dans l'exercice du métier, de le traiter, de le résoudre, guidées par un arrière-plan de représentations, d'expériences et d'attentes " (Payet, Sanchez-Mazas, Giuliani, Fernandez, 2011, p. 25). II s'agit de penser l'action technique - enseigner, faciliter l'acquisition des savoir-faire, promouvoir leur exercice - que l'outil viendra ensuite réaliser, renforcer, démultiplier.

Pour que les outils numériques contribuent à l'accessibilisation du système scolaire, il ne suffit pas d'équiper les établissements et de former aux outils, mais de travailler l'imbrication des outils aux gestes et aux postures qui rendent la pratique du métier d'enseignant opérante. Si du point de vue des gestes pris un à un, la dénomination geste générique (optimisation collective) ou geste spécifique (suppléance individuelle) peut être pertinente pour qualifier la nature et la destination des aides pédagogiques, elle ne l'est plus du point de vue du métier d'enseignant. Les gestes d'enseignement, planifiés ou produits dans l'action, se situent sur un continuum qui va du pôle de généricité à celui de spécificité. Les gestes sont plus ou moins génériques ou spécifiques, au sens où ils se rapprochent d'un pôle ou de l'autre, leur degré de généricité-spécificité oscillant tel un curseur entre les deux (Dunand, Feuilladieu, 2014, p. 121).

II ne suffit pas néanmoins de considérer les gestes principalement génériques comme des gestes à polarité d'accessibilité et les gestes principalement spécifiques comme des gestes à polarité de compensation. II est indispensable, pour disposer d'une typologie pertinente, de la doter du vecteur dynamique de l'opérationnalité inclusive. L'opérationnalité inclusive est le moteur de l'accessibilisation pédagogique et didactique du système scolaire. Elle s'appuie sur un rapport dialectique compensation-accessibilité, plus que sur un rapport d'opposition ou d'antinomie, mais elle implique l'enchâssement des aménagements capacitants dans l'action d'enseigner elle-même et ne peut être réduite à la simple transposition d'une adaptation ou d'une suppléance individuelle à une ou plusieurs autres personnes. L'analyse des exemples au prisme du modèle typologique des EIAH, montre l'évolution de certains outils d'une fonction à l'autre, selon l'usage qui en est fait. II 
nous semble ainsi heuristique de penser les outils numériques, notamment ceux prévus pour la scolarisation des élèves en situation de handicap ou de difficulté, plutôt dans une perspective d'usage inclusif que dans le cadre de l'intégration et de la normalisation individuelles, qui semble aujourd'hui encore largement majoritaire.

\section{Références}

Baron, G-L., Harrari, M. (2006). Les ressources informatisées en éducation. Entre invention, prescription et marchandisation, Médialog, 60, 36-41.

Barrette C. (2009). Métarecherche sur les effets de l'intégration des TIC en pédagogie collégiale, RITPU. Revue internationale des technologies en pédagogie universitaire, 6(2-3), 18-25. <https://www. erudit.org/fr/revues/ritpu/2009-v6-n23-ritpu1395677/1000008ar/> (consulté le 11 mai 2017).

Benoit, H. (2014a). Les dispositifs inclusifs: freins ou leviers pour l'évolution des pratiques. La nouvelle revue de l'adaptation et de la scolarisation, 65, 189-204.

Benoit, H. (2014b). Politiques publiques, professionnalités et langages: les maillons faibles de la chaîne inclusive. La nouvelle revue de l'adaptation et de la scolarisation, 67, 181-191.

Benoit, H. (2012a). Pluralité des acteurs et pratiques inclusives: les paradoxes de la collaboration. La nouvelle revue de l'adaptation et de la scolarisation, 57, 65-78.

Benoit, H. (2012b). Ressources numériques, didactisation et accessibilité: un modèle typologique. Éduquer - former, 44, 89-102.

Benoit, H. (2009). Pour une ingénierie de l'adaptation: des outils facilitateurs d'activités d'apprentissage. L'école numérique, 2, 32-33.

Benoit, H., Plaisance, É. (dir.). (2009). L'éducation inclusive en France et dans le monde, Hors série 5. La nouvelle revue de l'adaptation et de la scolarisation, $266 \mathrm{p}$.

Benoit, H. Sagot, J. (2008). L'apport des aides techniques à la scolarisation des élèves handicapés. La nouvelle revue de l'adaptation et de la scolarisation, 43, 19-26.

Benoit, H. (2007). Le plan incliné... La nouvelle revue de l'adaptation et de la scolarisation (Editorial), 39, 3-4.

Benoit, H. (2005). L'apprentissage de la lecture et l'appropriation de l'écrit dans I'éducation bilingue. La nouvelle revue de l'AIS, Hors série "Enseigner et apprendre en LSF, vers une éducation bilingue ", 45-52.

Bibeau, R. (2005). Les TIC à l'école: proposition de taxonomie et analyse des obstacles à leur intégration. Revue de I'EPI, wWw.epi.asso.fr/revue/articles/ a0511a.htm

Bonnéry, S. (2007). Comprendre l'échec scolaire: Élèves en difficultés et dispositifs pédagogiques. Paris: La Dispute.

Bril, B. et Roux, V. (2002). Le geste technique. Réflexions méthodologiques et anthropologiques. Paris: Érès. 
Cnesco. (2016). Conférence de comparaisons internationales. École inclusive pour les élèves en situation de handicap: accessibilité, réussite scolaire et parcours individuels (janvier 2016). http://www.cnesco.fr/fr/dossier-handicap/(consulté le 11 mai 2017).

Cresswell, R. (1975). Éléments d'ethnologie. Tome 2. Paris: A. Colin.

Creai Rhône-Alpes (2011). Handicap \& Scolarité 2011 - Les conditions de réussite des parcours de scolarisation - Compensation et accessibilité, quelle personnalisation? colloque, mercredi 23 mars 2011, École normale supérieure de Lyon.

D'Alessio, S. (2009). 30 ans d'Integrazione scolastica en Italie. Réflexions critiques et suggestions sur le développement de l'éducation inclusive en Italie. In $\mathrm{H}$. Benoit et É. Plaisance (dir.), L'éducation inclusive en France et dans le monde, Hors série 5, La nouvelle revue de l'adaptation et de la scolarisation, 35-50.

De Vries, E. (2001). Les logiciels d'apprentissage: panoplie ou éventail? Revue française de pédagogie, 137.

Dunand, D., Feuilladieu, S. (2014). Les aides pédagogiques aux élèves à besoins éducatifs particuliers: pratiques génériques ou spécifiques? La nouvelle revue de l'adaptation et de la scolarisation, 65, 113-124.

Ebersold, S. (2017). Trajectoires post-scolaires, transition juvénile et accessibilité: perspectives internationales. La nouvelle revue de l'adaptation et de la scolarisation, 77, 99-116.

Ebersold, S. (2017). L'éducation inclusive: privilège ou droit? Accessibilité et transition juvénile. Lycéen et après? Grenoble: PUG.

Ebersold S., Detraux J.-J (2013a), Scolarisation et besoin éducatif particuliers: enjeux conceptuels et méthodologiques d'une approche polycentrée, Alter. Revue européenne de recherche sur le handicap, 7(2) (numéro thématique), 102-115.

Ebersold, S. (2013b). De la transition comme référentiel analytique du devenir des élèves à BEP. La nouvelle revue de l'adaptation et de la scolarisation, 63, 15-28. Ebersold, S. (2009). Autour du mot "inclusion ", Recherche \& formation, 61, 71-83. https://rechercheformation.revues.org/522

Feuilladieu, S., Dunand, D. (2015). Gestes d'aide à l'étude en école élémentaire: variations observées auprès des élèves à besoins éducatifs particuliers. Symposium "Construire une communauté éducative pour tous: contributions des acteurs du terrain ". Rencontres internationales du réseau francophone de Recherche en Education et Formation. Montréal, Canada.

Flory, D., Benoit, H. (2010). Applications numériques, vie quotidienne des Sourds et apprentissages scolaires. La nouvelle revue de l'adaptation et de la scolarisation, 49, 153-161.

Foucault, M. (2011). Surveiller et punir. Gallimard, partie III-2, chapitre 2. (CEuvre originale publiée en 1975).

Fougeyrollas, P. (2010). La funambule, le fil et la toile. Transformations réciproques du sens du handicap. Québec: Les presses de l'université Laval.

Fougeyrollas, P. (2009). Entretien avec Patrick Fougeyrollas. Propos recueillis par Mouloud Boukala. La nouvelle revue de l'adaptation et de la scolarisation, 45, 165-174. 
Gardou, C., Poutoux V. (dir.). (2012). L'accessibilité à l'école, (dossier spécial), Éduquer et former, 44, $151 \mathrm{p}$.

Kirsch M. (2008). La pédagogie appuyée sur des preuves. Apprendre demain. Sciences cognitives et éducation à l'ère numérique. Paris: Hatier, 52-65.

Lahire, B. (2008). École et pratiques d'écriture, entre savoir et pouvoir. Rennes: Presses Universitaires de Rennes.

Larrouy, M. (2011), L'invention de l'accessibilité. Des politiques de transport des personnes handicapées aux politiques d'accessibilité de 1975 à 2005. Grenoble: Presses universitaires de Grenoble.

Marin, B. (2008). Produire un texte documentaire dans un environnement numérique en classe de Segpa: mobiliser des ressources informatives et cognitives. La nouvelle revue de l'adaptation et de la scolarisation, 42, 207-220.

Monette, S. et Bigras, M. (2008). La mesure des fonctions exécutives chez les enfants d'âge

préscolaire. Canadian Psychology, 49(4), 323-341. <https://Www.researchgate. net/publication/232489217_La_mesure_des_fonctions_executives_chez_les_ enfants_d\%27age_prescolaire_The_measurement_of_the_executive_functions_in_preschool_aged_children> (consulté le 19 mai 2017)

Orna, Observatoire des ressources numériques adaptées, <http://inshea.fr/fr/ content/orna-observatoire-des-ressources-numeriques-adaptees $>$

Payet, J.-P., Sanchez-Mazas, M., Giuliani, F.-E., et Fernandez, R. (2011). L'agir scolaire entre régulations et incertitudes. Vers une typologie des postures enseignantes de la relation à autrui. Éducation et sociétés, 27(1), 23-37.

Plaisance, É. (2013). De l'accessibilité physique à l'accessibilité pédagogique: vers un renouvellement des problématiques ? La nouvelle revue de l'adaptation et de la scolarisation, 63, 219-230.

Puimatto, G. (2004). Un historique, Les dossiers de l'ingénierie éducative, 46, www.cndp.fr/archivage/valid/55445/55445-8375-10296.pdf

Serres M. (2012). Petite poucette. Paris: Éditions Le Pommier.

Thibert R. (2012). Pédagogie + Numérique = Apprentissages 2.0. Dossier d'actualité Veille et Analyses, 79, novembre. <http://ife.ens-lyon.fr/vst/DA/detailsDossier.ph $p$ ?parent=accueil\&dossier $=79 \&$ lang $=f r>$

Velay, J.-L., Longchamp, M. (2005). Clavier ou stylo: comment écrire? Cerveau et psycho, 11, 3-7.

Velay, J.-L. (2003). De la plume au clavier: est-il encore utile d'enseigner l'écriture manuscrite? CNRS : Rapport de recherche. 